\title{
Late Recurrence of Hepatocellular Carcinoma in a Patient 10 Years after Liver Transplantation Unrelated to Transplanted Organ
}

\author{
Kornelia Morisson-Sarapak ${ }^{a}$ Maciej Wrzesiński ${ }^{a}$ Samir Zeair ${ }^{a}$ \\ Marta Wawrzynowicz-Syczewskab \\ aDepartment of General, Vascular and Transplantation Surgery of the Marie Curie \\ Hospital, Szczecin, Poland; b Department of Infectious Diseases, Hepatology and Liver \\ Transplantation, Pomeranian Medical University, Szczecin, Poland
}

\author{
Keywords \\ Hepatocarcinoma $\cdot$ Recurrence $\cdot$ Liver transplantation
}

\begin{abstract}
Liver transplantation (LTx) is an accepted method of hepatocellular carcinoma (HCC) treatment in cirrhotic patients; however, it has many limitations, and there is a substantial risk of recurrence. Most relapses occur within the first 2 posttransplant years. We aimed to present a late extrahepatic recurrence of HCC 10 years after LTX, and we discuss the possible risk factors and ways to improve transplantation results. A 68-year-old patient with liver cirrhosis and HCC on the background of chronic HCV and past HBV infection was transplanted urgently due to the rapid decompensation. Anti-HCV treatment before surgery was unsuccessful. Pretransplant computed tomography showed 1 focal $4.5 \mathrm{~cm}$ lesion consistent with HCC. Histopathology of the explanted organ showed 2 nodules outside the Milan criteria. Angioinvasion was not found. The patient achieved a sustained viral response to pegylated interferon and ribavirin 2 years post-LTx. Eight years were uneventful. CT of the abdomen performed occasionally was normal. Ten years after LTx, the patient unexpectedly presented with shortness of breath, fatigue, and weight loss. Two metastatic nodules of HCC in the lungs and pelvis were found. Although late HCC recurrence post-LTx is rare, it should be always considered, especially when risk factors such as viral infections and underestimation of tumor advancement were identified. We advocate that oncological surveillance of HCC relapse has to be continued during
\end{abstract}


Morisson-Sarapak et al.: Extrahepatic Recurrence of HCC in a Patient after Liver Transplantation

the whole posttransplant period. High AFP levels, the unfavorable neutrophil to lymphocyte ratio, and better estimation of primary tumor size seem to be useful in the identification of good candidates for transplantation.

(c) 2021 The Author(s).

Published by S. Karger AG, Basel

\section{Introduction}

One of the major indications for liver transplantation (LTx) is hepatocellular carcinoma (HCC). Patients undergoing LTx due to HCC are at increased risk of cancer recurrence. The risk of HCC recurrence after LTx depends on many factors, including tumor size, tumor stage, and angioinvasion. Moreover, patients undergoing chronic immunosuppressive treatment are at risk of developing de novo neoplasms - these are most often skin cancers, but there are also cases of de novo HCC; so far, several such cases have been described in the literature. Recurrent HCC can locate in various structures of the body; sometimes, it may not even appear in the liver. Therefore, it is important to perform comprehensive imaging control of liver recipients with a history of HCC, taking into account not only the abdominal organs but also the thorax and skeleton. Despite strictly defined rules of qualification for LTx, it is difficult to prevent HCC recurrence, the more so that no recommendations for screening tests for HCC have been developed.

\section{A Case Report}

The case concerns a 68-year-old patient transplanted in January 2010 at the department of general, vascular, and transplantation surgery of the Marie Curie Hospital, Szczecin, Poland, due to cirrhosis of the liver and coexisting primary liver cancer, both related to chronic HCV infection. Transplantation was performed urgently due to the high MELD score (26 points), which indicated severe decompensation of liver function.

A compensated cirrhosis type $\mathrm{C}$ was diagnosed in the patient in 2001 (stage A according to the Child-Turcotte-Pugh scale). The patient's history was also remarkable for cutaneous porphyria, type 2 diabetes, and arterial hypertension. During the first hospitalization in the hepatology department, laboratory tests revealed, apart from positive HCV RNA, features of a past HBV infection in the form of anti-HBc antibodies. The histopathological examination confirmed cirrhosis of the liver with mildly active inflammatory features. The patient underwent 6-month treatment with interferon and ribavirin, but virological response was not obtained. The patient remained under regular control of the hepatology outpatient clinic. In 2005, 48-week treatment with pegylated interferon and ribavirin was carried out with a good virological response at the end of therapy and recurrence of HCV infection after a few weeks. The first symptoms of cirrhosis decompensation such as ascites, jaundice, and coagulation disorders appeared in 2009. The MELD score at that time was 18. During hospitalization, ultrasound revealed a tumor in the right lobe of the liver with a diameter of approximately $4 \mathrm{~cm}$, confirmed by the abdominal CT scan. Due to the characteristic pattern of enhancement of a focal lesion on CT (enhancement in the arterial phase, Fig. 1, wash-out in the venous phase, Fig. 2), HCC was diagnosed. Tumor markers determined at that time were as follows: alphafetoprotein $2.7 \mathrm{IU} / \mathrm{mL}$, CA $19.9140 \mathrm{U} / \mathrm{mL}$, and CEA $10.1 \mu \mathrm{g} / \mathrm{mL}$. Due to the significant ascites, shown in Figures 1 and 2, the patient was disqualified from the tumor thermo-ablation procedure. After all the necessary examinations, envisaged by the liver transplantation qualification protocol, the patient was put on an active waiting list, and after ascites had been

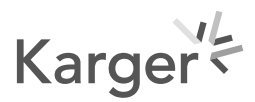


Fig. 1. Tumor enhancement in the arterial phase of the study.

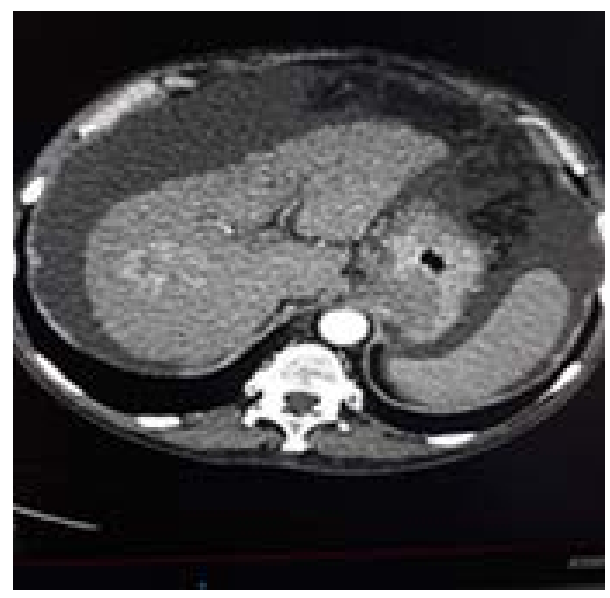

Fig. 2. Washout of contrast from the tumor in the venous phase.

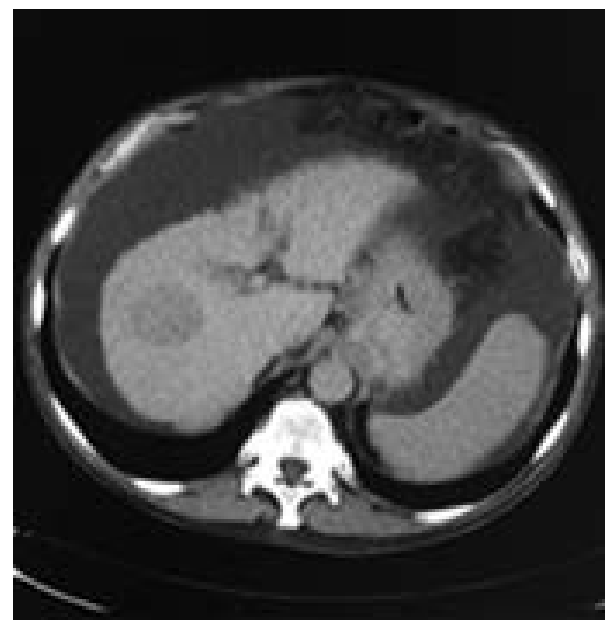

controlled in a stable state, he was discharged home. In January 2010, the patient was readmitted to the hepatology department due to hepatic encephalopathy in the course of decompensated cirrhosis. The MELD score increased to 26, and he was listed for urgent LTx. The course of the operation and the postoperative period as well as hospitalization in the surgery and transplantation department were uneventful. The patient in a good general condition was discharged home on day 17 with the recommendation of regular outpatient controls at the outpatient transplant clinic. After LTx, standard immunosuppressive treatment (tacrolimus, mycophenolate mofetil, and GSK for 12 weeks) and antiviral drugs such as lamivudine and acyclovir were implemented as a prophylaxis of HBV and CMV recurrence.

The histopathological examination of the grafted liver revealed bifocal G1 HCC with dimensions of $45 \mathrm{~mm}$ and $35 \mathrm{~mm}$, as well as micronodular cirrhosis and hemosiderosis.

In October 2010, the patient returned to the hepatology department due to a significant increase in the activity of liver enzymes, with suspected HCV reinfection in the transplanted liver, which was confirmed by histopathological examination and a significant increase in the serum viral load (HCV RNA) compared to the pretransplant viremia. Due to a significant leukopenia and a picture of mild hepatitis C (METAVIR A1F1), antiviral treatment was initially given up. Only after obtaining satisfactory blood counts, antiviral treatment with pegylated interferon and ribavirin was initiated and continued for 48 weeks with a good tolerance and till the end of treatment as well as sustained viral response (HCV RNA negative 
Fig. 3. Right lung tumor in arterial CT phase.

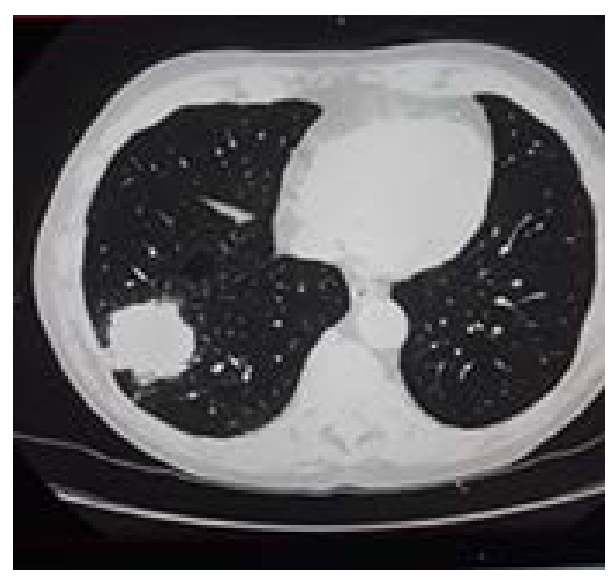

Fig. 4. Washout of contrast from the tumor.

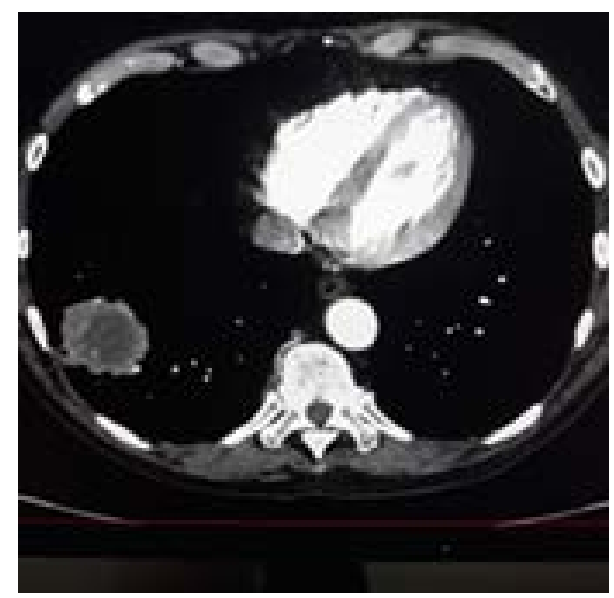

in 3 consecutive examinations and normalization of liver enzymes). Apart from a slight leukopenia, the patient remained without any laboratory abnormalities for the next several years.

In April 2013, the patient underwent comprehensive laboratory and imaging tests, including chest X-ray and ultrasound, in which no changes were shown. Stiffness of the liver, assessed by the elastography (Fibroscan) in 2016, was consistent with a mild fibrosis $-5.8 \mathrm{kPa}$ ). In August 2018, he came to his general practitioner due to shortness of breath and cough with expectoration of creamy secretion, as well as weight loss of about $4 \mathrm{~kg}$, complaints on getting tired more easily, and low-grade fever lasting from 3 months.

The chest X-ray showed a lesion of $50 \mathrm{~mm}$ in diameter in the right lung. The patient was admitted to the thoracic surgery and transplantation department, diagnostics was extended, a CT scan of the chest and abdominal cavity was performed, and the description of computed tomography in the fourth segment of the right lung revealed a $50-\mathrm{mm}$ tumor and a $13-\mathrm{mm}$ lymph node near the tumor (Fig. 3, 4). Liver changes were not found.

CT of the pelvis minor also uncovered a metastatic lesion, measuring $43 \times 12 \mathrm{~mm}$, in the left iliac bone. Bronchofiberoscopy was performed and no neoplastic cells were found in the cytological examination of the secretions; therefore, a fine-needle aspiration biopsy of the lesion in the lung was carried out, which confirmed the presence of HCC. The patient was eligible for palliative radiotherapy only.

In August 2019, he died of respiratory failure. 
Morisson-Sarapak et al.: Extrahepatic Recurrence of HCC in a Patient after Liver Transplantation

\section{Discussion}

Cirrhosis of the liver is the most important risk factor for the development of a primary liver cancer (HCC) and may be considered a precancerous condition. This is the case in the vast majority of patients with the underlying HCV infection. Dysplastic nodules undergo metaplasia and then neoplastic transformation, which results in the formation of HCC, which may initially be monofocal or multifocal, often extending over time.

At present, the most effective treatment is LTx, as it removes the direct cause of the cancer and the existing HCC. Unfortunately, the procedure of choice is burdened with a high rate of tumor recurrence; on average, $15-20 \%$ of patients transplanted for HCC within 2 years after transplantation [1]. However, a range of relapses can be found from $1.3 \%$ to $44.9 \%$, depending on the center [2].

Most relapses occur up to 2 years after LTx and most often affect the transplanted organ, less frequently the tumor metastasizes to the chest wall, lungs, bones, adrenal glands, and abdominal lymph nodes, or is generalized. In the case of late relapses, over 2 years after transplantation, and most often over 5 years, these proportions change, and extrahepatic recurrence or generalization of neoplastic disease occurs much more often [3-6]

A very rare situation in liver recipients is the de novo development of HCC; it may happen when HCC was not diagnosed in the recipient prior to LTx, neither on the basis of radiological examinations nor on the basis of the pathomorphological assessment of the explanted liver. HCC de novo appears at a similar time interval and localization as a late relapse.

With such a high percentage of HCC recurrences after LTx, it is important to identify the factors that predispose to relapse. It is not unusual in a cancer surgery that tumor size, or feature T, is a prognostic factor. During the initial period of liver transplantation program for HCC, the Milano criteria for the cutoff size of a tumor or tumors, eligible for Tx, were used strictly. However, in predicting the risk of HCC recurrence, very important pathomorphological features were observed, such as the presence of angioinvasion and a lower degree of tumor cell differentiation $[2,7]$.

Another factor, influencing recurrence, is the underestimation of tumor advancement in radiological examinations. Pathomorphological examinations of explanted livers sometimes reveal tumors, satellite nodules, and neoplastic changes in the local lymph nodes, not visualized in the imaging examinations. It is possible that the presence of micrometastases of a different biology causes a long-term dormancy, and the development of the disease extends over time [3]. The potential for relapse can be determined by the biochemical tests. A high pretransplant AFP level $(>1,000 \mathrm{IU} / \mathrm{mL})$ is a poor prognostic factor, as is the gradual build-up of AFP after LTx. More accurate in assessing the risk of HCC recurrence after transplantation may be the simultaneous analysis of 3 factors: the baseline AFP level, the neutrophil to lymphocyte ratio, and the size of the primary tumor [7, 8].

In general, risk factors for HCC recurrence after LTx can also be divided into 3 groups: tumor-related, patient-dependent, and treatment-dependent. Tumor-related factors include the aforementioned size, the occurrence of angioinvasion, the degree of differentiation of neoplastic cells, the initial level of AFP and its values after transplantation, and the tumor response to any possible bridging therapy [7]. Among the patient-dependent factors, the etiology of cirrhosis should be mentioned first with particular emphasis on visceral obesity (a factor important in the formation of many gastrointestinal cancers), untreated or ineffectively treated HCV infection, or past history of HBV infection. On the other hand, treatmentrelated factors can be divided into the pretransplant and posttransplant period. In the period before LTx, these include: percutaneous biopsy, transplant waiting time, application, and possible response to bridging therapy $[9,10]$. It seems that a better response to local treatment reduces the risk of HCC recurrence after transplantation, and a longer waiting period between

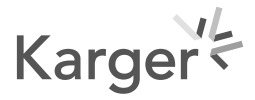


bridging and transplantation may allow the selection of patients with a less aggressive form of HCC. In the immediate perioperative period, the following should be mentioned: the donor's age, cold ischemia time and the surgical technique, and then the scheme of immunosuppression used [11].

Chronic HBV or HCV infection as an etiological factor of liver cirrhosis and recurrence of viral infection after LTx may also be considered as a possible de novo HCC factor, especially in cases where antiviral treatment was required after transplantation or the recipient redeveloped cirrhosis in the transplanted liver. Other risk factors may be transmission of tumor cells from a deceased donor or unrecognized recipient micrometastases. The possibility of transmitting specific genes is also taken into account, especially when a de novo cancer develops in a recipient of a living donation [12].

In all cases of early and late relapse and de novo HCC, men over 50 years were affected more frequently $[2,7,13]$. In our case, we can identify several factors that predispose to HCC recurrence after transplantation. These include chronic HCV infection as the etiology of cirrhosis, probable history of HBV infection earlier, antiviral treatment both before and after transplantation, and coexisting metabolic diseases (type 2 diabetes and cutaneous porphyria). Pathomorphological examination of the explanted liver confirmed the existence of a second neoplastic tumor (radiological underestimation). The existence of such an advanced tumor in the cirrhotic liver would be a factor that would preclude the eligibility for transplantation.

\section{Conclusions}

HCC recurrence after transplantation is a serious medical problem, and it is not only about the frequency of relapses but also about the fact that we are dealing with a patient after major abdominal surgery with altered vascular anatomy, especially arterial, who stays under constant immunosuppressive treatment. The options for locoregional surgical treatment of relapse are very limited, and emergency transplantation is unlikely. In the case of generalization of the disease, even systemic treatment remains questionable. Therefore, it seems that patients at risk of HCC recurrence should undergo more frequent radiological check-ups, not only in the first 2 years after LTx but also for a much longer period. The criteria for transplantation due to HCC remain debatable. It seems important to search for new HCC biomarkers that would enable to better identify patients at risk of HCC recurrence after transplantation. Work was in accordance with the principles of the Helsinki Convention.

\section{Statement of Ethics}

We received written informed consent from the patient's family to publish the manuscript containing information about the patient's medical history, including the results of diagnostic and imaging tests. Work was in accordance with the principles of the Helsinki Convention. The Bioethics Committee at the District Medical Chamber in Szczecin on December 3,2020 approved the observational retrospective study.

\section{Conflict of Interest Statement}

The authors have no conflicts of interest to declare.

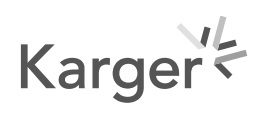




\section{Case Reports in Oncology}

Case Rep Oncol 2021;14:1754-1760

\begin{tabular}{l|l}
\hline DOI: 10.1159/000520535 & ○ 2021 The Author(s). Published by S. Karger AG, Basel
\end{tabular} www.karger.com/cro

Morisson-Sarapak et al.: Extrahepatic Recurrence of HCC in a Patient after Liver Transplantation

\section{Funding Sources}

We have no source of funding.

\section{Author Contributions}

Kornelia Morisson-Sarapak contributed to concept, obtaining data for work, sketch of work, a case report, translation, approval of the final version, control of intellectual content, and substantive evaluation. Maciej Wrzesiński contributed to concept, obtaining data for work, sketch of work, a case report, approval of the final version, control of intellectual content, and substantive evaluation. Samir Zeair contributed to concept, obtaining data for work, and approval of the final version. Marta Wawrzynowicz-Syczewska contributed to concept, obtaining data for work, approval of the final version, control of intellectual content, and revision of the manuscript.

\section{Data Availability Statement}

All patient data are available in the hospital database. If you wish to access patient data, please contact the corresponding author who will provide access to the data.

\section{References}

1 Bodzin AS, Lunsford KE, Markovic D, Harlander-Locke MP, Busuttil RW, Agopian VG. Predicting mortality in patients developing recurrent hepatocellular carcinoma after liver transplantation: impact of treatment modality and recurrence characteristics. Ann Surg. 2017;266:118-25.

2 Zhang JA, Kwee SA, Wong LL. Late recurrence of hepatocellular carcinoma after liver transplantation. Hepatoma Res. 2017;3:58-66.

3 Alshahrani AA, Ha SM, Hwang S, Ahn CS, Kim KH, Moon DB, et al. Clinical features and surveillance of very late hepatocellular carcinoma recurrence after liver transplantation. Ann Transplant. 2018;23:659-65.

4 de'Angelis N, Landi F, Carra MC, Azoulay D. Managements of recurrent hepatocellular carcinoma after liver transplantation: a systematic review. World J Gastroenterol. 2015;21:11185-98.

5 Ha TY, Hwang S, Ahn CS, Kim KH, Lee YJ, Moon DB, et al. Resection of metachronous adrenal metastasis after liver resection and transplantation for hepatocellular carcinoma. Dig Surg. 2014;31:428-35.

6 Xiang ZW, Sun L, Li GH, Maharjan R, Huang JH, Li CX. Progress in the treatment of pulmonary metastases after liver transplantation for hepatocellular carcinoma. World J Hepatol. 2015 Sep 18;7(20):2309-14.

7 Yao FY, Ferrell L, Bass NM, Watson JJ, Bacchetti P, VenookA, et al. Liver transplantation for hepatocellular carcinoma: expansion of the tumor size limits does not adversely impact survival. Hepatology. 2001;33:1394-403.

8 Shaw BI, Lucander A, Ravindra KV. Very late recurrence of hepatocellular carcinoma after orthotopic liver transplantation: presentation and management. Transplant Direct. 2019 Sep;5(9):e483.

$9 \mathrm{Au} \mathrm{KP}$, Chok KSH. Multidisciplinary approach for post-liver transplant recurrence of hepatocellular carcinoma: a proposed management algorithm. World J Gastroenterol. 2018 Dec 7;24(45):5081-94.

10 Hwang S, Kim YH, Kim DK, Ahn CS, Moon DB, Kim KH, et al. Resection of pulmonary metastases from hepatocellular carcinoma following liver transplantation. World J Surg. 2012;36:1592-1602.

11 Filgueria NA. Hepatocellular carcinoma recurrence after liver transplantation: risk factors, screening and clinical presentation. World J Hepatol. 2019;11(3):261-72.

12 Yu S, Guo H, Guo H, Zhuang L, Yu J, Yan S, et al. Case report of de novo hepatocellular carcinoma after living donor liver transplant. World J Surg Oncol. 2013;11:176.

13 Saab S, Zhou K, Chang EK, Busuttil RW. De novo hepatocellular carcinoma after liver transplantation. J Clin Transl Hepatol. 2015 Dec 28;3(4):284-7. 